International Journal of Pure and Applied Mathematics

Volume 109 No. 2 2016, 385-400

ISSN: 1311-8080 (printed version); ISSN: 1314-3395 (on-line version)

url: http://www.ijpam.eu

doi: $10.12732 /$ ijpam.v109i2.14

ijpam.eu

\title{
FIXED POINT THEOREMS FOR COMPATIBLE MAPPINGS AND ITS VARIANTS SATISFYING $(\psi-\phi)$-CONTRACTIVE CONDITIONS
}

\author{
Young Chel Kwun ${ }^{1}$, Poonam Nagpal ${ }^{2}$, Sanjay Kumar ${ }^{3}$, \\ Sudhir Kumar Garg ${ }^{4}$, Shin Min Kang 5 § \\ ${ }^{1}$ Department of Mathematics \\ Dong-A University \\ Busan, 49315, KOREA \\ ${ }^{2,3,4}$ Departement of Mathematics
}

Deenbandhu Chhotu Ram University of Science and Technology

Murthal, Sonepat 131039, Haryana, INDIA

${ }^{5}$ Department of Mathematics and RINS

Gyeongsang National University

Jinju, 52828, KOREA

Abstract: In this paper, we prove common fixed point theorems for compatible mappings and its variants satisfying $(\psi-\phi)$-contractive conditions in multiplicative metric spaces.

AMS Subject Classification: $47 \mathrm{H} 10,54 \mathrm{H} 25$

Key Words: multiplicative metric spaces, compatible mappings and its variants, altering distances

\section{Introduction and Preliminaries}

The applications of fixed point theory appears not only in many branches of mathematics but its application holds equally good in many branches of quantitative sciences such as economics, game theory, and computer sciences,

Received: July 8, 2016

Published: September 10, 2016

$\S$ Correspondence author (c) 2016 Academic Publications, Ltd.

url: www.acadpubl.eu 
etc. Fixed point theory has gained impetus, due to its wide range of applicability, to resolve diverse problems emanating from the theory of nonlinear differential equations and integral equations, game theory relevant to military, sports and medicine as well as economics. A metrical common fixed point theorem is broadly comprised of conditions on commutativity, continuity, completeness and contraction besides suitable containment of range of one map into the range of the other. For proving new results, the researchers of this domain are required to improve one or more of these conditions. The most famous result in this field is known as the Banach contraction principle.

In 1922, Banach [4] proved a fixed point theorem in complete metric spaces, which ensures under appropriate conditions, the existence and uniqueness of a fixed point. This result of Banach is known as Banach's fixed point theorem or Banach contraction principle. This theorem provides a technique for solving a variety of applied problems in mathematical sciences and engineering. The Banach contraction gave us many important theories like variational inequalities, optimization theory, and many computational theories. Due to the wide importance of the Banach contraction, many authors generalized it in several directions. Since the appearance of the Banach contraction mapping principle, a number of papers were dedicated to the improvement and generalization of that result. Most of these deal with the generalizations of the contractive condition in metric spaces.

Recently, Bashirov et al. [5] introduced the concept of multiplicative metric spaces to overcome the problem regarding the completeness of positive real numbers $\mathbb{R}_{+}$in the usual metric spaces and studied the concept of multiplicative calculus and proved the fundamental theorem of multiplicative calculus. In 2012, Florack and Assen [9] displayed the use of the concept of multiplicative calculus in biomedical image analysis.

In 2011, Bashirov et al. [6] exploit the efficiency of multiplicative calculus over the Newtonian calculus. They demonstrated that the multiplicative differential equations are more suitable than the ordinary differential equations in investigating some problems in various fields. They defined the multiplicative distance between two nonnegative real numbers as well as between two positive square matrices by using multiplicative absolute value function. This provides us basic tool in proving fixed points results in multiplicative metric spaces.

In 2012, Özavsar and Çevikel [13] gave the multiplicative metric spaces by remarking its topological properties, and introduced concept of multiplicative contraction mapping and proved some fixed point theorems of multiplicative contraction mappings on multiplicative spaces.

Bashirov et al. [5] introduced the concept of multiplicative metric spaces 
as follows:

Definition 1.1. Let $X$ be a nonempty set. A multiplicative metric is a mapping $d: X \times X \rightarrow \mathbb{R}_{+}$satisfying the following conditions:

(i) $d(x, y) \geq 1$ for all $x, y \in X$ and $d(x, y)=1$ if and only if $x=y$;

(ii) $d(x, y)=d(y, x)$ for all $x, y \in X$;

(iii) $d(x, y) \leq d(x, z) \cdot d(z, y)$ for all $x, y, z \in X$ (multiplicative triangle inequality).

Then the mapping $d$ together with $X$, that is, $(X, d)$ is a multiplicative metric space.

Example 1.2. ([13]) Let $\mathbb{R}_{+}^{n}$ be the collection of all $n$-tuples of positive real numbers. Let $d^{*}: \mathbb{R}_{+}^{n} \times \mathbb{R}_{+}^{n} \rightarrow \mathbb{R}$ be defined as follows:

$$
d^{*}(x, y)=\left|\frac{x_{1}}{y_{1}}\right|^{*} \cdot\left|\frac{x_{2}}{y_{2}}\right|^{*} \cdots\left|\frac{x_{n}}{y_{n}}\right|^{*},
$$

where $x=\left(x_{1}, \ldots, x_{n}\right), y=\left(y_{1}, \ldots, y_{n}\right) \in \mathbb{R}_{+}^{n}$ and $|\cdot|^{*}: \mathbb{R}_{+} \rightarrow \mathbb{R}_{+}$is defined by

$$
|a|^{*}= \begin{cases}a & \text { if } a \geq 1 \\ \frac{1}{a} & \text { if } a<1\end{cases}
$$

Then it is obvious that all conditions of a multiplicative metric are satisfied. Therefore $\left(\mathbb{R}_{+}^{n}, d^{*}\right)$ is a multiplicative metric space.

Example 1.3. ([15]) Let $d: \mathbb{R} \times \mathbb{R} \rightarrow[1, \infty)$ be defined as $d(x, y)=a^{|x-y|}$, where $x, y \in \mathbb{R}$ and $a>1$. Then $d$ is a multiplicative metric and $(\mathbb{R}, d)$ is a multiplicative metric space. We may call it usual multiplicative metric spaces.

Remark 1.4. We note that the Example 1.2 is valid for positive real numbers and Example 1.3 is valid for all real numbers.

Example 1.5. ([15]) Let $(X, d)$ be a metric space. Define a mapping $d_{a}$ on $X$ by

$$
d_{a}(x, y)=a^{d(x, y)}= \begin{cases}1 & \text { if } x=y \\ a & \text { if } x \neq y\end{cases}
$$

where $x, y \in X$ and $a>1$. Then $d_{a}$ is a multiplicative metric and $\left(X, d_{a}\right)$ is known as the discrete multiplicative metric space.

Example 1.6. ([1]) Let $X=C^{*}[a, b]$ be the collection of all real-valued multiplicative continuous functions on $[a, b] \subset \mathbb{R}_{+}$. Then $(X, d)$ is a multiplicative metric space with $d$ defined by $d(f, g)=\sup _{x \in[a, b]}\left|\frac{f(x)}{g(x)}\right|$ for arbitrary $f, g \in X$. 
Remark 1.7. ([15]) We note that multiplicative metrics and metric spaces are independent.

Indeed, the mapping $d^{*}$ defined in Example 1.2 is multiplicative metric but not metric as it does not satisfy triangular inequality. Consider

$$
d^{*}\left(\frac{1}{3}, \frac{1}{2}\right)+d^{*}\left(\frac{1}{2}, 3\right)=\frac{3}{2}+6=7.5<9=d^{*}\left(\frac{1}{3}, 3\right) .
$$

On the other hand the usual metric on $\mathbb{R}$ is not multiplicative metric as it doesnt satisfy multiplicative triangular inequality, since

$$
d(2,3) \cdot d(3,6)=3<4=d(2,6) .
$$

One can refer to [13] for detailed multiplicative metric topology.

Definition 1.8. Let $(X, d)$ be a multiplicative metric space. Then a sequence $\left\{x_{n}\right\}$ in $X$ is said to be

(1) a multiplicative convergent to $x$ if for every multiplicative open ball $B_{\epsilon}(x)=\{y \mid d(x, y)<\epsilon\}, \epsilon>1$, there exists $N \in \mathbb{N}$ such that $x_{n} \in B_{\epsilon}(x)$ for all $n \geq N$, that is, $d\left(x_{n}, x\right) \rightarrow 1$ as $n \rightarrow \infty$.

(2) a multiplicative Cauchy sequence if for all $\epsilon>1$, there exists $N \in \mathbb{N}$ such that $d\left(x_{n}, x_{m}\right)<\epsilon$ for all $m, n \geq N$, that is, $d\left(x_{n}, x_{m}\right) \rightarrow 1$ as $n, m \rightarrow \infty$.

(3) We call a multiplicative metric space complete if every multiplicative Cauchy sequence in it is multiplicative convergent to $x \in X$.

Remark 1.9. The set of positive real numbers $\mathbb{R}_{+}$is not complete according to the usual metric. Let $X=\mathbb{R}_{+}$and the sequence $\left\{x_{n}\right\}=\left\{\frac{1}{n}\right\}$. It is obvious $\left\{x_{n}\right\}$ is a Cauchy sequence in $X$ with respect to usual metric and $X$ is not a complete metric space, since $0 \notin \mathbb{R}_{+}$. In case of a multiplicative metric space, we take a sequence $\left\{x_{n}\right\}=\left\{a^{\frac{1}{n}}\right\}$, where $a>1$. Then $\left\{x_{n}\right\}$ is a multiplicative Cauchy sequence since for $n \geq m$,

$$
\begin{aligned}
d\left(x_{n}, x_{m}\right) & =\left|\frac{x_{n}}{x_{m}}\right|=\left|\frac{a^{\frac{1}{n}}}{a^{\frac{1}{m}}}\right|=\left|a^{\frac{1}{n}-\frac{1}{m}}\right| \\
& \leq a^{\frac{1}{m}-\frac{1}{n}}<a^{\frac{1}{m}}<\epsilon \quad \text { if } m>\frac{\log a}{\log \epsilon},
\end{aligned}
$$

where $|a|=\left\{\begin{array}{ll}a & \text { if } a \geq 1, \\ \frac{1}{a} & \text { if } a<1 .\end{array}\right.$ Also, $\left\{x_{n}\right\} \rightarrow 1$ as $n \rightarrow \infty$ and $1 \in \mathbb{R}_{+}$. Hence $(X, d)$ is a complete multiplicative metric space.

In 2012, Özavsar and Çevikel [13] gave the concept of multiplicative contraction mappings and proved some fixed point theorem of such mappings in a multiplicative metric space. 
Definition 1.10. Let $f$ be a mapping of a multiplicative metric space $(X, d)$ into itself. Then $f$ is said to be a multiplicative contraction if there exists a real number $\lambda \in[0,1)$ such that

$$
d(f x, f y) \leq d^{\lambda}(x, y) \quad \text { for all } x, y \in X .
$$

Altering distances is introduced by Khan et al. [12] in a metric space. These are control functions which alter the distance between two points in a metric space. Later on various author $([2,3,7,8,14,16,17]$ using altering distances proved common fixed point theorems.

In the similar mode, now we use the altering distances in multiplicative metric spaces. For this we define control functions as follows:

$\Psi=\{\psi \mid \psi:[1, \infty) \rightarrow[1, \infty)$ is continuous and non-decreasing satisfying $\psi(t)=1$ if and only if $t=1\}$.

$\Phi=\{\phi \mid \phi:[1, \infty) \rightarrow[1, \infty)$ is lower semi-continuous and discontinuous at $t=1, \phi(t)>1$ for all $t \geq 1\}$.

\section{Compatible Mappings and its Variants}

In 2015, Kang et al. [11] introduced the notion of compatible mappings and its variants as follows:

Definition 2.1. Let $f$ and $g$ be mappings of a multiplicative metric space $(X, d)$ into itself. Then $f$ and $g$ are called

(1) compatible if

$$
\lim _{n \rightarrow \infty} d\left(f g x_{n}, g f x_{n}\right)=1,
$$

whenever $\left\{x_{n}\right\}$ is a sequence in $X$ such that $\lim _{n \rightarrow \infty} f x_{n}=\lim _{n \rightarrow \infty} g x_{n}=t$ for some $t \in X$,

(2) compatible of type $(A)$ if

$$
\lim _{n \rightarrow \infty} d\left(f g x_{n}, g g x_{n}\right)=1 \text { and } \lim _{n \rightarrow \infty} d\left(g f x_{n}, f f x_{n}\right)=1,
$$

whenever $\left\{x_{n}\right\}$ is a sequence in $X$ such that $\lim _{n \rightarrow \infty} f x_{n}=\lim _{n \rightarrow \infty} g x_{n}=t$ for some $t \in X$,

(3) compatible of type $(B)$ if

$$
\lim _{n \rightarrow \infty} d\left(f g x_{n}, g g x_{n}\right) \leq\left[\lim _{n \rightarrow \infty} d\left(f g x_{n}, f t\right) \cdot \lim _{n \rightarrow \infty} d\left(f t, f f x_{n}\right)\right]^{1 / 2}
$$


and

$$
\lim _{n \rightarrow \infty} d\left(g f x_{n}, f f x_{n}\right) \leq\left[\lim _{n \rightarrow \infty} d\left(g f x_{n}, g t\right) \cdot \lim _{n \rightarrow \infty} d\left(g t, g g x_{n}\right)\right]^{1 / 2},
$$

whenever $\left\{x_{n}\right\}$ is a sequence in $X$ such that $\lim _{n \rightarrow \infty} f x_{n}=\lim _{n \rightarrow \infty} g x_{n}=t$ for some $t \in X$,

(4) compatible of type $(C)$ if

$\lim _{n \rightarrow \infty} d\left(f g x_{n}, g g x_{n}\right) \leq\left[\lim _{n \rightarrow \infty} d\left(f g x_{n}, f t\right) \cdot \lim _{n \rightarrow \infty} d\left(f t, f f x_{n}\right) \cdot \lim _{n \rightarrow \infty} d\left(f t, g g x_{n}\right)\right]^{1 / 3}$

and

$\lim _{n \rightarrow \infty} d\left(g f x_{n}, f f x_{n}\right) \leq\left[\lim _{n \rightarrow \infty} d\left(g f x_{n}, g t\right) \cdot \lim _{n \rightarrow \infty} d\left(g t, g g x_{n}\right) \cdot \lim _{n \rightarrow \infty} d\left(g t, f f x_{n}\right)\right]^{1 / 3}$,

whenever $\left\{x_{n}\right\}$ is a sequence in $X$ such that $\lim _{n \rightarrow \infty} f x_{n}=\lim _{n \rightarrow \infty} g x_{n}=t$ for some $t \in X$,

(5) compatible of type $(P)$ if

$$
\lim _{n \rightarrow \infty} d\left(f f x_{n}, g g x_{n}\right)=1
$$

whenever $\left\{x_{n}\right\}$ is a sequence in $X$ such that $\lim _{n \rightarrow \infty} f x_{n}=\lim _{n \rightarrow \infty} g x_{n}=t$ for some $t \in X$.

Also, Kang et al. [10] also introduced the notion of compatible mappings of type $(R)$, type $(K)$ and type $(E)$ as follows:

Definition 2.2. Let $f$ and $g$ be mappings of a multiplicative metric space $(X, d)$ into itself. Then $f$ and $g$ are called

(1) compatible of type $(R)$ if

$$
\lim _{n \rightarrow \infty} d\left(f g x_{n}, g f x_{n}\right)=1 \text { and } \lim _{n \rightarrow \infty} d\left(f f x_{n}, g g x_{n}\right)=1
$$

whenever $\left\{x_{n}\right\}$ is a sequence in $X$ such that $\lim _{n \rightarrow \infty} f x_{n}=\lim _{n \rightarrow \infty} g x_{n}=t$ for some $t \in X$

(2) compatible of type $(K)$ if

$$
\lim _{n \rightarrow \infty} d\left(f f x_{n}, g t\right)=1 \text { and } \lim _{n \rightarrow \infty} d\left(g g x_{n}, f t\right)=1,
$$

whenever $\left\{x_{n}\right\}$ is a sequence in $X$ such that $\lim _{n \rightarrow \infty} f x_{n}=\lim _{n \rightarrow \infty} g x_{n}=t$ for some $t \in X$, 
(3) compatible of type $(E)$ if

$$
\lim _{n \rightarrow \infty} f f x_{n}=\lim _{n \rightarrow \infty} f g x_{n}=g t \quad \text { and } \quad \lim _{n \rightarrow \infty} g g x_{n}=\lim _{n \rightarrow \infty} g f x_{n}=f t,
$$

whenever whenever $\left\{x_{n}\right\}$ is a sequence in $X$ such that $\lim _{n \rightarrow \infty} f x_{n}=\lim _{n \rightarrow \infty} g x_{n}=t$ for some $t \in X$.

Remark 2.3. Obviously, compatible mapping of type $(R)$ is also compatible mapping and compatible mapping of type $(P)$.

Proposition 2.4. ([11]) Let $f$ and $g$ be mappings of a multiplicative metric space $(X, d)$ into itself. Assume that $f$ and $g$ are compatible or compatible of type $(A)$ or of type $(B)$ or of type $(C)$ or of type $(P)$ and $f t=g t$ for some $t \in X$. Then $f g t=f f t=g g t=g f t$.

Proposition 2.5. ([10]) Let $f$ and $g$ be mappings of a multiplicative metric space $(X, d)$ into itself. Assume that $f$ and $g$ are compatible of type $(R)$ or of type $(K)$ or of type $(E)$ and $f t=g t$ for some $t \in X$. Then $f g t=f f t=$ $g g t=g f t$.

\section{Main Results}

Now, we prove the following results for compatible mappings.

Theorem 3.1. Let $A, B, S$ and $T$ be mappings of a complete multiplicative metric space $(X, d)$ into itself satisfying

$$
\begin{gathered}
A(X) \subset T(X) \text { and } B(X) \subset S(X), \\
\psi(d(A x, B y)) \leq \frac{\psi(M(x, y))}{\phi(N(x, y))}
\end{gathered}
$$

for all $x, y \in X$ with $x \neq y$, where $\psi \in \Psi, \phi \in \Phi$,

$$
\begin{gathered}
M(x, y)=\max \left\{d(S x, T y),(d(S x, A x) \cdot d(T y, B y))^{1 / 2},\right. \\
\left.(d(S x, B y) \cdot d(T y, A x))^{1 / 2}\right\}
\end{gathered}
$$

and

$$
N(x, y)=\min \{d(S x, T y), d(S x, A x), d(T y, B y)\},
$$

(C3) One of the subspace $A X$ or $B X$ or $S X$ or $T X$ is closed.

Assume that the pairs $A, S$ and $B, T$ are compatible. Then $A, B, S$ and $T$ have a unique common fixed point in $X$. 
Proof. Let $x_{0} \in X$ be arbitrary point. Since $A(X) \subset T(X)$ and $B(X) \subset$ $S(X)$, there exist a point $x_{1} \in X$ such that $A x_{0}=T x_{1}$ and for this point $x_{1} \in X$, there exists a point $x_{2} \in X$ such that $B x_{1}=S x_{2}$. Continuing this process, we can define a sequence $\left\{y_{n}\right\}$ in $X$ such that

$$
y_{2 n+1}=A x_{2 n}=T x_{2 n+1}, \quad y_{2 n+2}=B x_{2 n+1}=S x_{2 n+2} \quad \text { for } n \geq 0 .
$$

Now assume that $y_{n} \neq y_{n+1}$ for all $n \in \mathbb{N} \cup\{0\}$.

Putting $x=x_{2 n}$ and $y=x_{2 n+1}$ in (C2), we have

$$
\psi\left(d\left(A x_{2 n}, B x_{2 n+1}\right)\right) \leq \frac{\psi\left(M\left(x_{2 n}, x_{2 n+1}\right)\right)}{\phi\left(N\left(x_{2 n}, x_{2 n+1}\right)\right)},
$$

where

$$
\begin{aligned}
& M\left(x_{2 n}, x_{2 n+1}\right) \\
& =\max \left\{d\left(S x_{2 n}, T x_{2 n+1}\right),\left(d\left(S x_{2 n}, A x_{2 n}\right) \cdot d\left(T x_{2 n+1}, B x_{2 n+1}\right)\right)^{1 / 2},\right. \\
& \left.\quad\left(d\left(S x_{2 n}, B x_{2 n+1}\right) \cdot d\left(T x_{2 n+1}, A x_{2 n}\right)\right)^{1 / 2}\right\}
\end{aligned}
$$

and

$$
\begin{aligned}
& N\left(x_{2 n}, x_{2 n+1}\right) \\
& =\min \left\{d\left(S x_{2 n}, T x_{2 n+1}\right), d\left(S x_{2 n}, A x_{2 n}\right), d\left(T x_{2 n+1}, B x_{2 n+1}\right)\right\} .
\end{aligned}
$$

Then using multiplicative triangular inequality, we have

$$
\begin{aligned}
& M\left(x_{2 n}, x_{2 n+1}\right) \\
& =\max \left\{d\left(y_{2 n}, y_{2 n+1}\right), d\left(y_{2 n}, y_{2 n+1}\right) \cdot d\left(y_{2 n+1}, y_{2 n+2}\right)\right)^{1 / 2}, \\
& \left.\quad\left(d\left(y_{2 n}, y_{2 n+2}\right) \cdot d\left(y_{2 n+1}, y_{2 n+1}\right)\right)^{1 / 2}\right\} \\
& \leq \max \left\{d\left(y_{2 n}, y_{2 n+1}\right), d\left(y_{2 n}, y_{2 n+1}\right) \cdot d\left(y_{2 n+1}, y_{2 n+2}\right)\right)^{1 / 2}, \\
& \left.\quad\left(d\left(y_{2 n}, y_{2 n+1}\right) \cdot d\left(y_{2 n+1}, y_{2 n+2}\right)\right)^{1 / 2}\right\}
\end{aligned}
$$

and

$$
N\left(x_{2 n}, x_{2 n+1}\right)=\min \left\{d\left(y_{2 n}, y_{2 n+1}\right), d\left(y_{2 n}, y_{2 n+1}\right), d\left(y_{2 n+1}, y_{2 n+2}\right)\right\}
$$

If $d\left(y_{2 n}, y_{2 n+1}\right)<d\left(y_{2 n+1}, y_{2 n+2}\right)$, then we get

$$
M\left(x_{2 n}, x_{2 n+1}\right) \leq d\left(y_{2 n+1}, y_{2 n+2}\right)
$$

and

$$
N\left(x_{2 n}, x_{2 n+1}\right)=d\left(y_{2 n}, y_{2 n+1}\right)>1
$$


Hence $\phi\left(N\left(x_{2 n}, x_{2 n+1}\right)\right)>1$.

From (3.1), we have

$$
\begin{aligned}
\psi\left(d\left(y_{2 n+1}, y_{2 n+2}\right)\right) & \leq \frac{\psi\left(M\left(x_{2 n}, x_{2 n+1}\right)\right)}{\phi\left(N\left(x_{2 n}, x_{2 n+1}\right)\right)} \\
& <\psi\left(M\left(x_{2 n}, x_{2 n+1}\right)\right)
\end{aligned}
$$

Since $\psi$ is non-decreasing, we have

$$
d\left(y_{2 n+1}, y_{2 n+2}\right) \leq M\left(x_{2 n}, x_{2 n+1}\right) .
$$

Again using (3.2) and (3.3), we have

$$
M\left(x_{2 n}, x_{2 n+1}\right)=d\left(y_{2 n+1}, y_{2 n+2}\right) .
$$

From (3.1) and the property of $\psi$ and $\phi$, we have

$$
\psi\left(d\left(y_{2 n+1}, y_{2 n+2}\right)\right) \leq \frac{\psi\left(d\left(y_{2 n+1}, y_{2 n+2}\right)\right)}{\phi\left(N\left(x_{2 n}, x_{2 n+1}\right)\right)}<\psi\left(d\left(y_{2 n+1}, y_{2 n+2}\right)\right)
$$

which is a contradiction. Thus we have

$$
d\left(y_{2 n+1}, y_{2 n+2}\right) \leq d\left(y_{2 n}, y_{2 n+1}\right)
$$

Now we have

$$
M\left(x_{2 n}, x_{2 n+1}\right)=d\left(y_{2 n}, y_{2 n+1}\right), \quad N\left(x_{2 n}, x_{2 n+1}\right)=d\left(y_{2 n+1}, y_{2 n+2}\right) .
$$

Using (3.4) in (3.1), we have

$$
\psi\left(d\left(y_{2 n+1}, y_{2 n+2}\right)\right) \leq \frac{\psi\left(d\left(y_{2 n}, y_{2 n+1}\right)\right)}{\phi\left(N\left(x_{2 n}, x_{2 n+1}\right)\right)} \leq \psi\left(d\left(y_{2 n}, y_{2 n+1}\right)\right) .
$$

Therefore $\left\{d\left(y_{2 n}, y_{2 n+1}\right)\right\}$ is a non-increasing sequence of non-negative real numbers and it is bounded below by 1 , there exists a number $r \geq 1$ such that

$$
\lim _{n \rightarrow \infty} d\left(y_{2 n}, y_{2 n+1}\right)=r \geq 1 .
$$

Assume that $r>1$. Letting $n \rightarrow \infty$, we have

$$
\lim _{n \rightarrow \infty} \psi\left(d\left(y_{2 n+1}, y_{2 n+2}\right)\right) \leq \frac{\lim _{n \rightarrow \infty} \psi\left(d\left(y_{2 n}, y_{2 n+1}\right)\right)}{\lim _{n \rightarrow \infty} \phi\left(N\left(x_{2 n}, x_{2 n+1}\right)\right)}
$$


which implies that

$$
\psi(r) \leq \frac{\psi(r)}{\lim _{n \rightarrow \infty} \phi\left(N\left(x_{2 n}, x_{2 n+1}\right)\right)}<\psi(r),
$$

which is a contradiction by property of $\phi$.

Indeed, we obtain

$$
\lim _{n \rightarrow \infty} N\left(x_{2 n}, x_{2 n+1}\right)=\lim _{n \rightarrow \infty} d\left(x_{2 n+1}, x_{2 n+2}\right)=r>1,
$$

which implies that

$$
\lim _{n \rightarrow \infty} \phi\left(N\left(x_{2 n}, x_{2 n+1}\right) \geq \phi(r)>1 .\right.
$$

Therefore, we have

$$
\lim _{n \rightarrow \infty} d\left(y_{2 n}, y_{2 n+1}\right)=1 .
$$

Putting $x=x_{2 n+1}$ and $y=x_{2 n+2}$ in $(C 2)$ and proceeding as above, we obtain

$$
\lim _{n \rightarrow \infty} d\left(y_{2 n+1}, y_{2 n+2}\right)=1
$$

Therefore, for all $n \in \mathbb{N} \cup\{0\}$, we have

$$
\lim _{n \rightarrow \infty} d\left(y_{n}, y_{n+1}\right)=1 . .
$$

Now, we prove that $\left\{y_{n}\right\}$ is a multiplicative Cauchy sequence. For this it is enough to show that the subsequence $\left\{y_{2 n}\right\}$ is a multiplicative Cauchy sequence. To the contrary, suppose that $\left\{y_{2 n}\right\}$ is not a multiplicative Cauchy sequence. Then there exists an $\epsilon>1$ and the sequence of natural numbers $\{2 n(k)\}$ and $\{2 m(k)\}$ with $2 n(k)>2 m(k) \geq 2 k$ for $k \in \mathbb{N}$ such that

$$
d\left(y_{2 m(k)}, y_{2 n(k)}\right) \geq \epsilon .
$$

Corresponding to $2 m(k)$, we can choose $2 n(k)$ to be the smallest satisfying (3.6). Then we have

$$
d\left(y_{2 m(k)}, y_{2 n(k)-2}\right)<\epsilon .
$$

Putting $x=x_{2 m(k)}$ and $y=x_{2 n(k)-1}$ in $(C 2)$, where $k \in \mathbb{N}$,

$$
\begin{aligned}
\psi\left(d\left(y_{2 m(k)+1}, y_{2 n(k)}\right)\right) & =\psi\left(d\left(A x_{2 m(k)}, B x_{2 n(k)-1}\right)\right) \\
& \leq \frac{\psi\left(M\left(x_{2 m(k)}, x_{2 n(k)-1}\right)\right)}{\phi\left(N\left(x_{2 m(k)}, x_{2 n(k)-1}\right)\right)}
\end{aligned}
$$


where

$$
\begin{aligned}
M( & \left.x_{2 m(k)}, x_{2 n(k)-1}\right) \\
= & \max \left\{d\left(S x_{2 m(k)}, T x_{2 n(k)-1}\right),\right. \\
& \left(d\left(S x_{2 m(k)}, A x_{2 m(k)}\right) \cdot d\left(T x_{2 n(k)-1}, B x_{2 n(k)-1}\right)\right)^{1 / 2} \\
& \left.\left(d\left(S x_{2 m(k)}, B x_{2 n(k)-1}\right) \cdot d\left(T x_{2 n(k)-1}, A x_{2 m(k)}\right)\right)^{1 / 2}\right\} \\
= & \max \left\{\left(d\left(y_{2 m(k)}, y_{2 n(k)-1}\right),\left(d\left(y_{2 m(k)}, y_{2 m(k)+1}\right) \cdot d\left(y_{2 n(k)-1}, y_{2 n(k)}\right)\right)^{1 / 2},\right.\right. \\
& \left.\left(d\left(y_{2 m(k)}, y_{2 n(k)}\right) \cdot d\left(y_{2 n(k)-1}, y_{2 m(k)+1}\right)\right)^{1 / 2}\right\}
\end{aligned}
$$

and

$$
\begin{aligned}
& N\left(x_{2 m(k)}, x_{2 n(k)-1}\right) \\
& =\min \left\{\left(d\left(y_{2 m(k)}, y_{2 n(k)-1}\right), d\left(y_{2 m(k)}, y_{2 m(k)+1}\right), d\left(y_{2 n(k)-1}, y_{2 n(k)}\right)\right\}\right.
\end{aligned}
$$

Using the multiplicative triangular inequality, we have

$$
\begin{aligned}
\epsilon & \leq d\left(y_{2 m(k)}, y_{2 n(k)}\right) \\
& \leq d\left(y_{2 m(k)}, y_{2 n(k)-2}\right) \cdot d\left(y_{2 n(k)-2}, y_{2 n(k)-1}\right) \cdot d\left(y_{2 n(k)-1}, y_{2 n(k)}\right) .
\end{aligned}
$$

Letting $k \rightarrow \infty$ and using (3.5), we get

$$
\lim _{k \rightarrow \infty} d\left(y_{2 m(k)}, y_{2 n(k)}\right)=\epsilon
$$

Again, we have

$$
d\left(y_{2 n(k)-1}, y_{2 m(k)}\right) \leq d\left(y_{2 n(k)}, y_{2 n(k)-1}\right) \cdot d\left(y_{2 n(k)}, y_{2 m(k)}\right)
$$

and

$$
d\left(y_{2 m(k)}, y_{2 n(k)}\right) \leq d\left(y_{2 n(k)}, y_{2 n(k)-1}\right) \cdot d\left(y_{2 n(k)-1}, y_{2 m(k)}\right) .
$$

Letting $k \rightarrow \infty$ and using (3.5), (3.7), we have

$$
\lim _{k \rightarrow \infty} d\left(y_{2 n(k)-1}, y_{2 m(k)}\right)=\epsilon
$$

Also, by multiplicative triangular inequality, we have

$$
d\left(y_{2 m(k)+1}, y_{2 n(k)}\right) \leq d\left(y_{2 m(k)+1}, y_{2 m(k)}\right) \cdot d\left(y_{2 m(k)}, y_{2 n(k)}\right)
$$

and

$$
d\left(y_{2 m(k)}, y_{2 n(k)}\right) \leq d\left(y_{2 m(k)}, y_{2 m(k)+1}\right) \cdot d\left(y_{2 m(k)+1}, y_{2 n(k)}\right)
$$


Letting $k \rightarrow \infty$ and using (3.7), we have

$$
\lim _{k \rightarrow \infty} d\left(y_{2 m(k)+1}, y_{2 n(k)}\right)=\epsilon
$$

Again, we have

$$
d\left(y_{2 n(k)-1}, y_{2 m(k)+1}\right) \leq d\left(y_{2 n(k)-1}, y_{2 n(k)}\right) \cdot d\left(y_{2 n(k)}, y_{2 m(k)+1}\right)
$$

and

$$
d\left(y_{2 m(k)+1}, y_{2 n(k)}\right) \leq d\left(y_{2 m(k)+1}, y_{2 n(k)-1}\right) \cdot d\left(y_{2 n(k)-1}, y_{2 n(k)}\right)
$$

Letting $k \rightarrow \infty$ and using (3.5), (3.9), we have

$$
\lim _{k \rightarrow \infty} d\left(y_{2 m(k)+1}, y_{2 n(k)-1}\right)=\epsilon
$$

From (3.5), (3.7), (3.8) and (3.10), we have

$$
\lim _{k \rightarrow \infty} M\left(x_{2 m(k)}, x_{2 n(k)-1}\right)=\epsilon \quad \text { and } \quad \lim _{k \rightarrow \infty} N\left(x_{2 m(k)}, x_{2 n(k)-1}\right)=1 .
$$

Letting $k \rightarrow \infty$, we get

$$
\psi(\epsilon) \leq \frac{\psi(\epsilon)}{\lim _{n \rightarrow \infty} \phi\left(N\left(x_{2 m(k)}, x_{2 n(k)-1}\right)\right)} .
$$

Using discontinuity of $\phi$ at $t=1$, since $\lim _{n \rightarrow \infty} \phi\left(N\left(x_{2 m(k)}, x_{2 n(k)-1}\right)\right)>\phi(1)>1$, we get contradiction. Hence $\left\{y_{n}\right\}$ is a multiplicative Cauchy sequence. Since $(X, d)$ is complete, $\left\{y_{n}\right\}$ converges to a point $z \in X$. Consequently, the subsequences also converges to $z \in X$. Thus

$$
A x_{2 n} \rightarrow z, \quad T x_{2 n+1} \rightarrow z, \quad B x_{2 n+1} \rightarrow z, \quad S x_{2 n} \rightarrow z .
$$

Suppose that $S X$ is closed. Then there exists $v \in X$ such that $z=S v$.

Now, suppose that $z \neq A v$. Putting $x=v$ and $y=x_{2 n+1}$ in $(C 2)$, we get

$$
\psi\left(d\left(A v, B x_{2 n+1}\right)\right) \leq \frac{\psi\left(M\left(v, x_{2 n+1}\right)\right)}{\phi\left(N\left(v, x_{2 n+1}\right)\right)},
$$

where

$$
\begin{gathered}
M\left(v, x_{2 n+1}\right)=\max \left\{d\left(S v, T x_{2 n+1}\right),\left(d(S v, A v) \cdot d\left(T x_{2 n+1}, B x_{2 n+1}\right)\right)^{1 / 2}\right. \\
\left.\left(d\left(S v, B x_{2 n+1}\right) \cdot d\left(T x_{2 n+1}, A v\right)\right)^{1 / 2}\right\}
\end{gathered}
$$


and

$$
N\left(v, x_{2 n+1}\right)=\min \left\{d\left(S v, T x_{2 n+1}\right), d(S v, A v), d\left(T x_{2 n+1}, B x_{2 n+1}\right)\right\} .
$$

Letting $n \rightarrow \infty$ and using $z=S v$, we have

$$
\begin{aligned}
& \lim _{n \rightarrow \infty} M\left(v, x_{2 n+1}\right) \\
& =\max \left\{d(S v, z),(d(S v, A v) \cdot d(z, z))^{1 / 2},(d(S v, z) \cdot d(z, A v))^{1 / 2}\right\} \\
& =d^{1 / 2}(z, A v)
\end{aligned}
$$

and

$$
\lim _{n \rightarrow \infty} N\left(v, x_{2 n+1}\right)=1
$$

So, we have

$$
\psi\left(d(A v, z) \leq \frac{\psi\left(d^{1 / 2}(z, A v)\right)}{\lim _{n \rightarrow \infty} \phi\left(N\left(v, x_{2 n+1}\right)\right)} .\right.
$$

Using discontinuity of $\phi$ at $t=1$, we obtain

$$
\psi(d(A v, z))<\psi\left(d^{1 / 2}(z, A v)\right),
$$

which is a contradiction as $\psi$ is non-decreasing. Therefore, we have $d(z, A v)=$ 1. and hence $A v=z=S v$. So $A$ and $S$ have a coincidence point $v$. By Proposition 2.4, $A$ and $S$ commute at coincidence point. Therefore, $A S v=S A v$ and hence $A z=S z$.

Next, we show that $A z=S z=z$. Putting $x=z$ and $y=x_{2 n+1}$ in $(C 2)$, we get

$$
\psi\left(d\left(A z, B x_{2 n+1}\right)\right) \leq \frac{\psi\left(M\left(z, x_{2 n+1}\right)\right)}{\phi\left(N\left(z, x_{2 n+1}\right)\right)},
$$

where

$$
\begin{gathered}
M\left(z, x_{2 n+1}\right)=\max \left\{d\left(S z, T x_{2 n+1}\right),\left(d(S z, A z) \cdot d\left(T x_{2 n+1}, B x_{2 n+1}\right)\right)^{1 / 2},\right. \\
\left.\left(d\left(S z, B x_{2 n+1}\right) \cdot d\left(T x_{2 n+1}, A z\right)\right)^{1 / 2}\right\}
\end{gathered}
$$

and

$$
N\left(z, x_{2 n+1}\right)=\min \left\{d\left(S z, T x_{2 n+1}\right), d(S z, A z), d\left(T x_{2 n+1}, B x_{2 n+1}\right)\right\}
$$

Letting $n \rightarrow \infty$ and using $A z=S z$, we have

$$
\lim _{n \rightarrow \infty} M\left(z, x_{2 n+1}\right)=d(S z, z)
$$


and

$$
\lim _{n \rightarrow \infty} N\left(z, x_{2 n+1}\right)=1
$$

So we have

$$
\psi\left(d(S z, z) \leq \frac{\psi(d(S z, z))}{\lim _{n \rightarrow \infty} \phi\left(N\left(z, x_{2 n+1}\right)\right)} .\right.
$$

Using discontinuity of $\phi$ at $t=1$, we obtain

$$
\psi(d(S z, z))<\psi(d(S z, z))
$$

which is a contradiction. Therefore $d(S z, z)=1$ and hence $A z=S z=z$.

Similarly, we can show that $T z=B z=z$. Hence $S z=A z=T z=B z=z$. Therefore $A, B, S$ and $T$ have a common fixed point in $X$

Similarly, we can also complete the proof when $A X$ or $B X$ or $T X$ is closed.

Finally, we show that $z$ is a unique common fixed point of $A, B, S$ and $T$. Suppose that $z$ and $w(z \neq w)$ are also fixed points of $A, B, S$ and $T$.

Putting $x=z$ and $y=w$ in $(C 2)$, we have

$$
\begin{aligned}
\psi(d(z, w)) & =\psi(d(A z, B w)) \\
& \leq \frac{\psi(M(z, w))}{\phi(N(z, w))}<\psi(d(z, w))
\end{aligned}
$$

where

$$
\begin{aligned}
M(z, w)= & \max \left\{d(S z, T w),(d(S z, A z) \cdot d(T w, B w))^{1 / 2}\right. \\
& \left.(d(S z, B w) \cdot d(T w, A z))^{1 / 2}\right\} \\
= & d(z, w)
\end{aligned}
$$

and

$$
\begin{aligned}
N(z, w) & =\min \{d(S z, T w), d(S z, A z), d(T w, B w)\} \\
& =1,
\end{aligned}
$$

which is a contradiction. Hence $z=w$. Therefore $A, B, S$ and $T$ have a unique common fixed point in $X$. This completes the proof.

In Theorem 3.1, if we put $\psi(t)=t$, we obtain the following corollary.

Corollary 3.2. Let $A, B, S$ and $T$ be mappings of a complete multiplicative metric space $(X, d)$ into itself satisfying $\left(C_{1}\right),\left(C_{3}\right)$ and

$$
d(A x, B y) \leq \frac{M(x, y)}{\phi(N(x, y))}
$$


for all $x, y \in X$ with $x \neq y$, where $\phi \in \Phi$,

$$
\begin{gathered}
M(x, y)=\max \left\{d(S x, T y),(d(S x, A x) \cdot d(T y, B y))^{1 / 2},\right. \\
\left.(d(S x, B y) \cdot d(T y, A x))^{1 / 2}\right\}
\end{gathered}
$$

and

$$
N(x, y)=\min \{d(S x, T y), d(S x, A x), d(T y, B y)\} .
$$

Assume that the pairs $A, S$ and $B, T$ are compatible. Then $A, B, S$ and $T$ have a unique common fixed point in $X$.

In Theorem 3.1 and Corollary 3.2, if we put $S=T=I$, the identity mapping, we obtain the following corollaries.

Corollary 3.3. Let $A$ and $B$ be mappings of a complete multiplicative metric space $(X, d)$ into itself satisfying

$$
\psi(d(A x, B y)) \leq \frac{\psi(M(x, y))}{\phi(N(x, y))}
$$

for all $x, y \in X$ with $x \neq y$, where $\psi \in \Psi, \phi \in \Phi$,

$$
M(x, y)=\max \left\{d(x, y),(d(x, A x) \cdot d(y, B y))^{1 / 2},(d(x, B y) \cdot d(y, A x))^{1 / 2}\right\}
$$

and

$$
N(x, y)=\min \{d(x, y), d(x, A x), d(y, B y)\}
$$

Then $A$ and $B$ have a unique common fixed point in $X$.

Corollary 3.4. Let $A$ and $B$ be mappings of a complete multiplicative metric space $(X, d)$ into itself satisfying

$$
d(A x, B y) \leq \frac{M(x, y)}{\phi(N(x, y))}
$$

for all $x, y \in X$ with $x \neq y$, where $\phi \in \Phi$,

$$
M(x, y)=\max \left\{d(x, y),(d(x, A x) \cdot d(y, B y))^{1 / 2},(d(x, B y) \cdot d(y, A x))^{1 / 2}\right\}
$$

and

$$
N(x, y)=\min \{d(x, y), d(x, A x), d(y, B y)\}
$$

Then $A$ and $B$ have a unique common fixed point in $X$.

Next, we prove the following theorem for compatible mappings of type $(A)$ or of type $(B)$ or of type $(C)$ or of type $(P)$. 
Theorem 3.5. Let $A, B, S$ and $T$ be mappings of a complete multiplicative metric space $(X, d)$ into itself satisfying $\left(C_{1}\right),\left(C_{2}\right)$ and $\left(C_{3}\right)$.

Assume that the pairs $A, S$ and $B, T$ are compatible of type $(A)$ or of type $(B)$ or of type $(C)$ or of type $(P)$. Then $A, B, S$ and $T$ have a unique common fixed point in $X$.

Proof. Proceeding from the proof as in Theorem 3.1, $A$ and $S$ have a coincidence point $v$. Thus we have $z=A v=S v$. Using Proposition 2.4, we have $A S v=S S v=S A v=A A v$ and hence $A z=S z$. The rest parts of the proof follows from Theorem 3.1.

Finally, we give the following theorem for compatible mappings of type $(R)$ or of type $(K)$ or of type $(E)$.

Theorem 3.6. Let $A, B, S$ and $T$ be mappings of a complete multiplicative metric space $(X, d)$ into itself satisfying $\left(C_{1}\right),\left(C_{2}\right)$ and $\left(C_{3}\right)$.

Assume that the pairs $A, S$ and $B, T$ are compatible of type $(R)$ or of type $(K)$ or of type $(E)$. Then $A, B, S$ and $T$ have a unique common fixed point in $X$.

Proof. Proceeding from the proof as in Theorem 3.1, $A$ and $S$ have a coincidence point $v$. Thus we have $z=A v=S v$. Using Proposition 2.5, we have $A S v=S S v=S A v=A A v$ and hence $A z=S z$. The rest parts of the proof follows from Theorem 3.1.

\section{Acknowledgments}

This work was supported by the Dong-A University research fund.

\section{References}

[1] M. Abbas, B. Ali, Y.I. Suleiman, Common fixed points of locally contractive mappings in multiplicative metric spaces with application, Int. J. Math. Math. Sci., 2015 (2015), Article ID 218683, 7 pages. doi: 10.1155/2015/218683.

[2] M. Abbas, D. Đorić, Common fixed point theorem for four mappings satisfying generalized weak contractive condition, Filomat 24 (2010), 1-10. doi: 10.2298/FIL1002001A

[3] M. Abbas, M.A. Khan, Common fixed point theorem of two mappings satisfying a generalized weak contractive condition, Int. J. Math. Math. Sci., 2009 (2009), Article ID 131068, 9 pages. doi: 10.1155/2009/131068 
[4] S. Banach, Sur les opérations dans les ensembles abstracts et leur application aux équations intégrales, Fund. Math., 3 (1922), 133-181.

[5] A.E. Bashirov, E.M. Kurplnara, A. Ozyapici, Multiplicative calculus and its applicatiopns, J. Math. Anal. Appl., 337 (2008), 36-48. doi: 10.1016/j.jmaa.2007.03.081

[6] A.E. Bashirov, E. Msrl, Y. Tandoğdu, A. Özyapc, On modeling with multiplicative differential equations, Appl. Math. J. Chinese Univ. Ser. B, 26 (2011), 425-438. doi: 10.1007/s11766-011-2767-6

[7] P.N. Dutta, B.S. Choudhury, A generalization of contraction principle in metric spaces, Fixed Point Theory Appl., (2008) (2008), Article ID 406368, 8 pages. doi: $10.1155 / 2008 / 406368$

[8] D. Đorić, Common fixed point for generalized $(\psi, \phi)$-weak contractions, Appl. Math. Lett., 22 (2009), 1896-1900. doi: 10.1016/j.aml.2009.08.001

[9] L. Florack, H. V. Assen, Multiplicative calculus in biomedical image analysis, J. Math. Imaging Vision, 42 (2012), 64-75. doi: 10.1007/s10851-011-0275-1

[10] S.M. Kang, P. Kumar, S. Kumar, Common fixed points for compatible mappings of types in multiplicative metric spaces, Int. J. Math. Anal., 9 (2015), 1755-1767. doi: 10.12988/ijma.2015.53104

[11] S.M. Kang, P. Kumar, S. Kumar, P. Nagpal, S.K Garg, Common fixed points for compatible mappings and its variants in multiplicative metric spaces, Int. J. Pure Appl. Math., 102 (2015), 383-406. doi: 10.12732/ijpam.v102i2.14

[12] M.S. Khan, M. Swaleh, S. Sessa, Fixed point theorems by altering distances between the points, Bull. Austral. Math. Soc., 30 (1984), 1-9.

[13] M. Özavsar, A.C. Çevikel, Fixed points of multiplicative contraction mappings on multiplicative metric spaces, ArXiv:1205.5131v1 [math.GM] (2012), 14 pages.

[14] B.E. Rhoades, Some theorems on weakly contractive maps, Nonlinear Anal., 47 (2001), 2683-2693. doi: 10.1016/S0362-546X(01)00388-1

[15] M. Sarwar, R. Badshah-e, Some unique fixed point theorems in multiplicative metric space, ArXiv:1410.3384v2 [matn.GM] (2014), 19 pages.

[16] K.P.R. Sastry, S.V.R. Naidu, G.V.R. Babu, G.A. Naidu, Generalization of common fixed point theorems for weakly commuting mappings by altering distances, Tamkang J. Math., 31 (2000), 243-250.

[17] Q. Zhang, Y. Song, Fixed point theory for generalized $\psi$-weak contractions, Appl. Math. Lett., 22 (2009), 75-78. doi: 10.1016/j.aml.2008.02.007 
